\title{
Creating long-lived neutral-atom traps in a cryogenic environment
}

\author{
P. A. Willems and K. G. Libbrecht \\ Norman Bridge Laboratory of Physics, 12-33 California Institute of Technology, Pasadena, California 91125
}

(Received 11 July 1994; revised manuscript received 29 September 1994)

\begin{abstract}
We describe techniques for creating long-lived magneto-optical and magnetostatic traps for neutral atoms. These traps exist in a sealed cryogenic environment with a temperature near $4 \mathrm{~K}$, where the background gas pressure can be extremely low. To date we have observed cesium magneto-optical traps with background-limited lifetimes in excess of $1 \mathrm{~h}$, and magnetostatic traps with lifetimes of nearly 10 min. From these observations we use the known He-Cs van der Waals collision cross section to infer typical background gas pressures in our apparatus below $4 \times 10^{-12}$ Torr. With hardware improvements we expect this pressure can be made much lower, extending the magnetostatic-trap lifetimes one to two orders of magnitude. Furthermore, with a cryogenic system one can use superconducting magnets and SQUID detectors to trap and to nondestructively sense spin-polarized atoms. With superconducting microstructures one can achieve very large magnetic-field gradients and curvatures, as high as $\sim 10^{6} \mathrm{G} / \mathrm{cm}$ and $\sim 10^{9} \mathrm{G} / \mathrm{cm}^{2}$, respectively, for use in magnetic and magneto-optical traps.
\end{abstract}

PACS number(s): 33.80.Ps

\section{INTRODUCTION}

The ability to produce easily copious numbers of ultracold neutral atoms using laser cooling and trapping techniques has opened many new avenues in atomic physics. Recent technical advances in this area include different types of magneto-optical traps (MOT's) (e.g., the "standard" quadrupole MOT [1], the "dark-spot" MOT [2], the rectified-dipole MOT [3]), several geometries of magnetic-dipole traps (e.g., the Ioffe-baseball [4,5] and ac dipole traps $[6,7])$, microwave traps [8], far-off-resonance dipole traps [9], trapping in optical crystals [10], atomic fountains [11], atom optics, atom interferometry [12], and numerous others. Applications and potential applications of these new technologies include investigations of novel atomic collisions in traps, such as associative ionization [13] and photoassociation processes [14], improved atomic clocks [15], an improved measurement of the fine-structure constant [16], the production of weakly interacting Bose-Einstein condensed gases [17], and others.

In many of these areas, the loss of trapped atoms due to collisions with background gas atoms is an important consideration. For example, one of the most promising routes for achieving Bose-Einstein condensation of alkali-metal atoms is via evaporative cooling in a magnetic trap. Since the trap densities and atomic velocities are typically both low, the time scale for evaporative cooling can be several minutes or longer [17]. For these time scales background collisions in traditional vacuum systems present a serious limitation [5].

We have constructed a general-purpose apparatus for producing laser cooled and trapped atoms in a cryogenic environment, where the background gas densities can be very low, leading to longer trap lifetimes. We report here some details of our experimental apparatus and our first observations of long-lived traps with this system.

\section{EXPERIMENTAL APPARATUS}

Figure 1 shows a schematic diagram of our cryogenic apparatus, which consists essentially of a cooled chamber with ample optical access and a shutter to admit a beam of thermal cesium atoms. By pumping on the helium exhaust of the continuous-flow cryostat the cold-finger temperature was run at typically 3.6 to $4.2 \mathrm{~K}$. Allowing for various heat loads on the system and the finite thermal

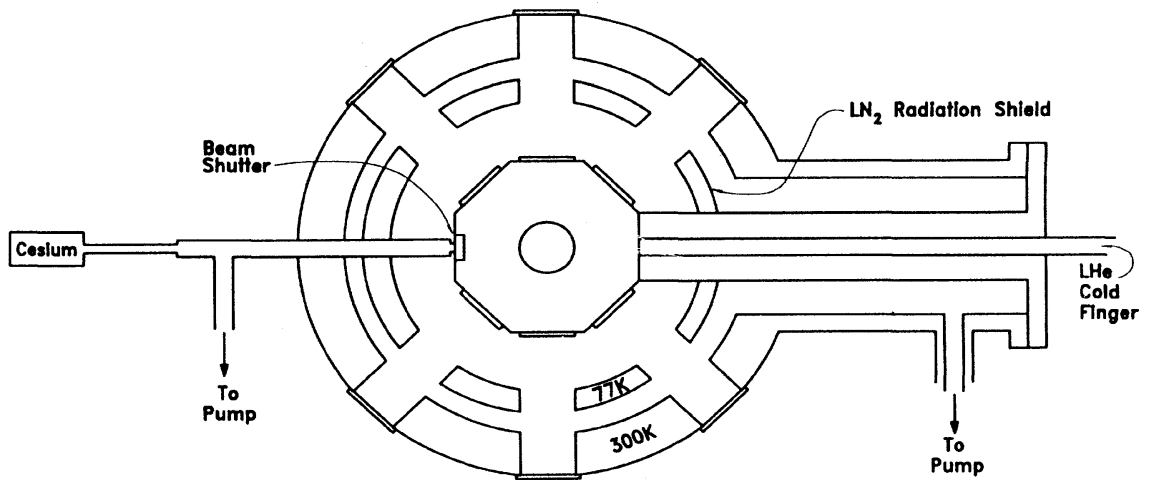

FIG. 1. Apparatus used for laser cooling and trapping neutral cesium atoms in a cryogenic environment. The central chamber, covered with optical windows, is cooled to $\sim 4 \mathrm{~K}$ by a continuous-flow cryostat. The diameter of the outer vacuum chamber is $20.3 \mathrm{~cm}$. 
conduction between the cold finger and the inner chamber, we estimate that the chamber temperature was typically between 6 and $9 \mathrm{~K}$. Normal coils outside the vacuum chamber and/or superconducting coils inside the cryogenic region provide the necessary magnetic fields for atom trapping. A vacuum pump attached to the inner vacuum chamber typically produces a pressure of $\sim 10^{-7}$ Torr in the room-temperature part, and a vacuum is maintained in the outer chamber to prevent condensation and excessive heat load to the system. During data collection the beam shutter is closed, isolating the cryogenic part of the inner vacuum chamber.

The expected background gas density in a sealed dark chamber cooled to $4 \mathrm{~K}$ can be extremely low, assuming that the walls of the chamber are not saturated with helium. Pressures as low $5 \times 10^{-17}$ Torr have been reported [18], and under ideal conditions at $4 \mathrm{~K}$ one theoretically expects an essentially perfect vacuum [19]. Light incident on the inner walls of the chamber can significantly increase the background pressure, due to light-induced desorption of helium from the cryogenic surfaces [10]. Because of this, we may expect pressures below $\sim 10^{-12}$ Torr to be difficult to attain in the presence of scattered light from atom-trapping laser beams.

\section{EXPERIMENTAL RESULTS}

\section{A. Magneto-optical traps}

In an investigation of long-lived magneto-optical traps, we used external magnetic-field coils to generate a weak quadrupole field in the inner chamber, with a typical axial gradient $B^{\prime} \approx 10 \mathrm{G} / \mathrm{cm}$, and loaded the trap directly from an uncooled cesium beam. Once the trap was loaded, the beam shutter was closed and the trap fluorescence monitored as a function of time. Figure 2 shows data with a fairly high initial trap population, where the decay is clearly dominated by collisions between trapped cesium

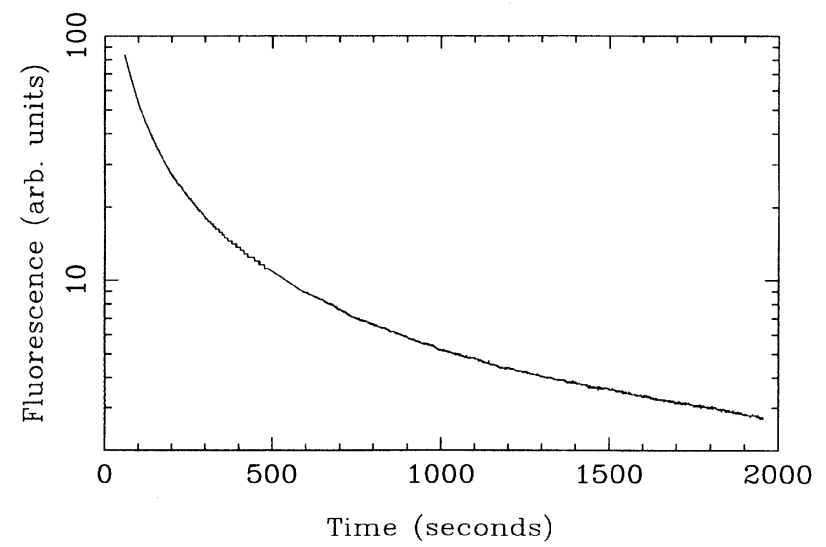

FIG. 2. Magneto-optical trap fluorescence as a function of time, with approximately 70000 atoms initially loaded into the trap, and axial field gradient $B^{\prime}=6.7 \mathrm{G} / \mathrm{cm}$. Note that the observed trap decay is from Cs-Cs collisions between trapped atoms, with essentially no losses from collisions with background gas atoms. From data like these we infer $\alpha^{-1} \gtrsim 60 \mathrm{~min}$ for deep traps. atoms. After correcting for a measured residual trap loading rate (see below), we modeled the trap loss with $\dot{n}=-\alpha n-\beta n^{2}$, where $n$ is the density of trapped atoms (assumed proportional to fluorescence). We measured $\alpha \approx 0$ and values of $\beta$ ranging over $(2-20) \times 10^{-11} \mathrm{~cm}^{3} / \mathrm{s}$, the latter consistent with values reported elsewhere [21]. With very low trap populations we measured $n / \dot{n}$ as high as 50 min still consistent with no losses from background collisions. From these data we can place a lower limit of $\alpha^{-1} \gtrsim 60 \mathrm{~min}$ for a fairly deep MOT. We expect $\alpha$ to be extremely small not only because of the low number of background gas molecules, but also because these molecules (predominantly hydrogen and helium) are light and moving slowly; thus a typical $\mathrm{He}-\mathrm{Cs}$ or $\mathrm{H}_{2}$-Cs elastic collision does not impart enough kinetic energy to a cesium atom to eject it from the MOT.

With low trap populations we also observed an unusual trap loading rate with the cesium beam blocked, of typically $\sim 0.2$ atoms $/ \mathrm{sec}$ at field gradient of $10 \mathrm{G} / \mathrm{cm}$. This was measured by observations of MOT fluorescence with no initial trap loading from the thermal beam $[n(t=0)=0]$. The loading rate was not well correlated with the tightness of the beam shutter seal, suggesting that the loading was not due to leakage around the beam shutter. We believe the loading is due to cesium atoms weakly bound to the inner surface of the cryogenic chamber, which are subsequently released by lightinduced desorption. A brief "bake" of the chamber at 25 $K$ with the beam shutter open, to release the adsorbed helium and/or hydrogen molecules, resulted in a reduction of the trap loading rate by a factor of approximately 5.

\section{B. Magnetostatic traps}

We also investigated the lifetimes of quadrupole magnetostatic atom traps in our apparatus, using a pair of superconducting coils to generate the required magnetic fields. After first loading a MOT with an axial field gradient of $50 \mathrm{G} / \mathrm{cm}$, the cesium beam shutter was closed and the repumping laser (tuned to the $F=3 \rightarrow F^{\prime}=4$ transition) was blocked, so the MOT trapping lasers (tuned just below $F=4 \rightarrow F^{\prime}=5$ ) subsequently pumped all the atoms into the $F=3$ ground state. A few milliseconds later, the trapping lasers were also blocked. Approximately $10 \%$ of the initial MOT population was thus trapped in a quadrupole magnetostatic trap (MST). After a waiting period $T$ with both lasers blocked, the trapping and repumping lasers were then unblocked in reverse order and the MOT reformed. By measuring the MOT fluorescence as a function of $T$ we determined the MST decay time. MST decays at higher field gradients were measured by first forming the MST at $50 \mathrm{G} / \mathrm{cm}$, then ramping up the field (while the lasers were blocked), waiting a time $T$, ramping the field back down to $50 \mathrm{G} / \mathrm{cm}$, and reforming the MOT. Figure 3(a) shows MST decay measurements at $50 \mathrm{G} / \mathrm{cm}$, and Fig. 3(b) shows MST decay times as a function of an axial field gradient. We found that MST decay times depended to some degree on the recent history of the vacuum system, and the best results were obtained only with frequent $25 \mathrm{~K}$ bakes to re- 
move adsorbed gas. From this it appears that our MST lifetimes are currently limited in part by the pressure of the room-temperature part of the inner vacuum chamber, typically $10^{-7}$ Torr.

We expect the MST losses to be dominated by collisions with background gas, which we assume is predominantly helium. This gives a conservative estimate of the vacuum quality, since $\mathrm{He}$ is heavier and has a slightly stronger van der Waals interaction with Cs than does $\mathbf{H}_{2}$. For small-angle scattering we can write the He-Cs collisional rate equation

$$
\begin{aligned}
\frac{1}{N} \frac{d N}{d t} & \simeq 2 \pi \int \sigma(\theta, v) \theta d \theta d n(v) v \\
& \equiv n_{0} \int \widetilde{\sigma}(E) d E,
\end{aligned}
$$

where $N$ is the number of trapped Cs atoms, $\sigma(\theta, v)$ is the
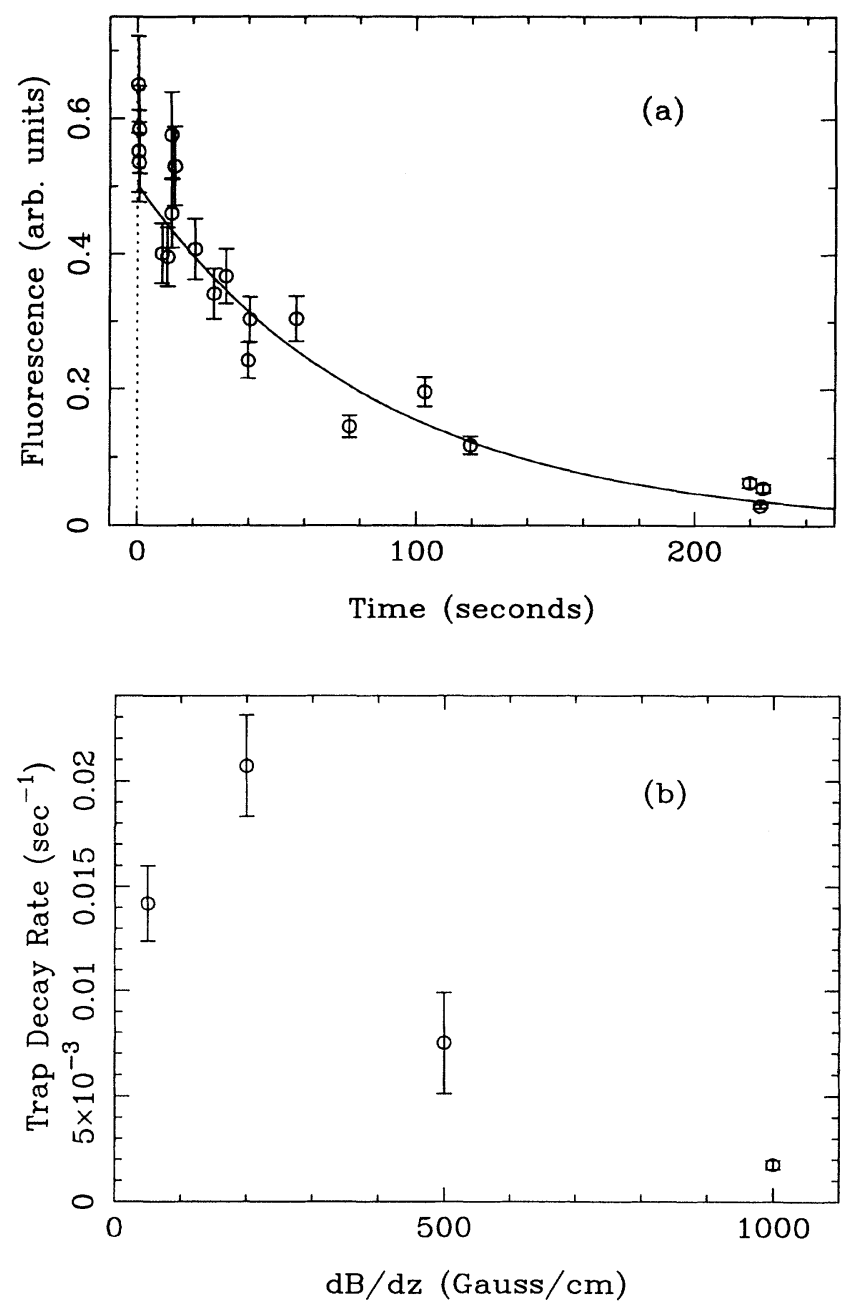

FIG. 3. (a) Observed magneto-static trap decay, for a quadrupole trap with axial field gradient $B^{\prime}=50 \mathrm{G} / \mathrm{cm}$. The scatter in the points at $t=0$ reflects the variations in trap loading rate. The curve through the points is a fit exponential decay $y=A e^{-\alpha t}$. (b) Trap decay constant $\alpha$ as a function of a field gradient. The scattering theory described in the text results in a much weaker field dependence than observed.

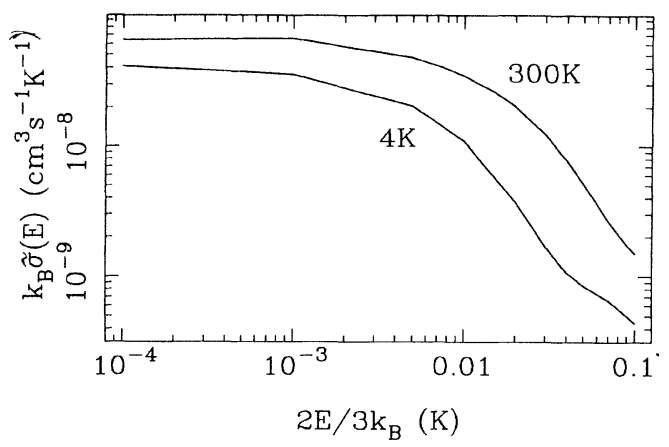

FIG. 4. Differential scattering cross section $\widetilde{\sigma}(E)$ described in the text, in temperature units with $E=k_{B} / 2 T$. Here we have integrated over a Boltzmann distribution of $\mathrm{He}$ atoms at temperatures of 4 and $300 \mathrm{~K}$.

van der Waals cross section $[22,23]$ as a function of the $\mathrm{He}$ recoil angle $\theta$ and $\mathrm{He}$ initial velocity $v, E \simeq m_{\mathrm{He}}^{2} v^{2} \theta^{2} / 2 m_{\mathrm{Cs}}$ is the Cs kinetic energy after a collision, $n_{0}$ is the total $\mathrm{He}$ density, and $d n(v)$ is the Boltzmann velocity distribution with $\int d n=n_{0}$. Figure 4 shows the calculated $\widetilde{\sigma}(E)$ for background $\mathrm{He}$ at temperatures of 4 and $300 \mathrm{~K}$, using a He-Cs van der Waals coefficient [24] $C_{6}=3.2 \times 10^{-78} \mathrm{~J} \mathrm{~m}^{6}$. Figure 5 shows $\int_{E_{0}}^{\infty} \widetilde{\sigma}(E) d E$, which gives the trap loss rate as a function of trap depth $E_{0}$.

We estimate the trap depth for our coil configuration to be $\sim 20 B^{\prime} \mu \mathrm{K}$, where $B^{\prime}$ is the axial field gradient in $\mathrm{G} / \mathrm{cm}$. This gives a trap decay which decreases slightly as $B^{\prime}$ increases, in contrast to the observations in Fig. 3(b). We believe this discrepancy may be due to additional MST losses arising from scattered laser light in the trapping region. Even a very low level of scattered light, primarily from the repumping laser, would be sufficient to optically pump atoms into untrapped magnetic sublevels at the observed rates. Further, the optical pumping losses are expected to have a strong (albeit somewhat complex) dependence on $B^{\prime}$, which may explain the observations. Using the measured MST decay time at 1

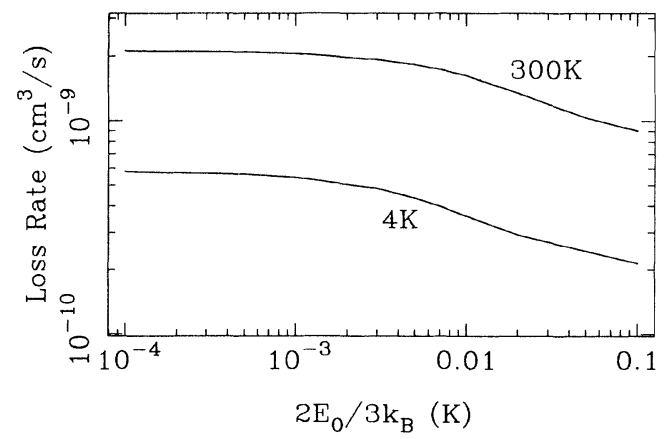

FIG. 5. Integrated cross section $\int_{E_{0}}^{\infty} \widetilde{\sigma}(E) d E$. Multiplying this by the total $\mathrm{He}$ background density $n_{0}$ gives the trap loss rate $N^{-1} d N / d t$ as a function of trap depth $E_{0}$. 
$\mathrm{kG} / \mathrm{cm}$ to set an upper limit on the background pressure gives $P<4 \times 10^{-12}$ Torr. It should be noted that the construction of the cryogenic chamber and the difficulty in determining pressure at cryogenic temperatures made a measurement of the vacuum pressure by other means impractical.

\section{DISCUSSION}

In summary, we have demonstrated long-lived magneto-optical and magnetostatic cesium atom traps in a cryogenic apparatus. Long-lived magnetostatic traps have previously been demonstrated with sodium [25] and hydrogen [26], both using cryogenic chambers to achieve very low background gas densities. The present apparatus differs from these others in having optical access along more than a single axis in order to exploit fully laser cooling and trapping technologies. Although the trap loss rates we have observed are good but not extraordinary, there is much room to improve our present apparatus. For instance, by further cooling the cryogenic vacuum chamber, improving the room temperature part of the inner chamber vacuum from $\sim 10^{-7}$ to $\sim^{-9}$ Torr or lower, and eliminating scattered laser light, we believe we can realize magnetostatic-trap losses one to two orders of magnitude lower than we have demonstrated.

Another advantage of the cryogenic environment worth noting is the ability to use superconducting struc- tures for producing large magnetic-field gradients and curvatures for atom trapping. We have used a pair of superconducting coils $\sim 0.5 \mathrm{~cm}$ in radius to produce quadrupole fields with gradients as high as $50 \mathrm{kG} / \mathrm{cm}$, and it should be possible to push this up to $\sim 10^{6} \mathrm{G} / \mathrm{cm}$ with more careful design. Spin-polarized magnetostatic traps, such as the Ioffe trap, have no magnetic-field gradient at their centers, and it is the curvature (second derivative) of the field which determines their trapping force. The curvature scales as $B^{\prime \prime} \sim \rho / S$, where $\rho$ is the critical current density and $S$ is the characteristic size of the coil structure. Using microfabrication techniques to construct microscopic superconducting magnetic-field coils, it may be possible to achieve field curvatures of $10^{9} \mathrm{G} / \mathrm{cm}^{2}$, and spin-polarized MST frequencies above $k_{B} T_{\text {recoil }} / h$, where $T_{\text {recoil }}$ is the recoil limit temperature for laser cooling [27]. Finally, we note that SQUID detectors offer a means to nondestructively sense the presence of trapped spin-polarized atoms [28].

\section{ACKNOWLEDGMENTS}

We thank H. J. Kimble and members of the California Institute of Technology Quantum Optics group for frequent technical and scientific assistance, and also Richard Boyd and Gane Wong for useful discussions. This work was supported by the AT\&T Special Grants Program, the Hale Foundation, and the California Institute of Technology.
[1] E. L. Raab, M. Prentiss, A. Cable, S. Chu, and D. E. Pritchard, Phys. Rev. Lett. 59, 2631 (1987).

[2] W. Ketterle, K. B. Davis, M. A. Joffe, A. Martin, and D. E. Pritchard, Phys. Rev. Lett. 70, 2253 (1993).

[3] O. Emile, F. Bardou, C. Salomon, Ph. Laurent, A. Nadir, and A. Clairon, Europhys. Lett. 20, 687 (1992).

[4] T. Bergeman, G. Erez, and H. J. Metcalf, Phys. Rev. A 35, 1535 (1987).

[5] C. R. Monroe, E. A. Cornell, C. A. Sackett, C. J. Myatt, and C. E. Wieman, Phys. Rev. Lett. 70, 414 (1993).

[6] E. A. Cornell, C. Monroe, and C. E. Wieman, Phys. Rev. Lett. 67, 2439 (1991).

[7] R. V. E. Lovelace, C. Mehanian, T. J. Tommila, and D. M. Lee, Nature (London) 318, 30 (1985).

[8] R. J. C. Spreeuw, C. Gerz, L. S. Goldner, W. D. Phillips, S. L. Rolston, C. I. Westbrook, M. W. Reynolds, and I. F. Silvera, Phys. Rev. Lett. 72, 3162 (1994).

[9] J. D. Miller, R. A. Cline, and D. J. Heinzen, Phys. Rev. A 47, R4567 (1993).

[10] G. Grynberg, B. Lounis, P. Verkerk, J. Y. Courtois, and C. Salomon, Phys. Rev. Lett. 70, 2249 (1993); A Hemmerich, C. Zimmermann, and T. W. Hänsch, Europhys. Lett. 22, 89 (1993).

[11] M. A. Kasevich, E. Riis, S. Chu, and R. G. DeVoe, Phys. Rev. Lett. 63, 612 (1989).

[12] D. W. Keith, C. R. Ekstrom, Q. A. Turchette, and D. E. Pritchard, Phys. Rev. Lett. 66, 2693 (1991); M. Kasevich and S. Chu, ibid. 67, 181 (1991).

[13] P. L. Gould, P. D. Lett. P. S. Julienne, W. D. Phillips, H. R. Thorsheim, and J. Weiner, Phys. Rev. Lett. 60, 788
(1988).

[14] J. D. Miller, R. A. Cline, and D. J. Heinzen, Phys. Rev. Lett. 71, 2204 (1993).

[15] K. Gibble and S. Chu, Phys. Rev. Lett. 70, 1771 (1993); Metrologia 29, 201 (1992).

[16] D. S. Weiss, B. C. Young, and S. Chu, Phys. Rev. Lett. 70, 2706 (1993).

[17] E. Tiesinga, A. J. Moerdijk, B. J. Verhaar, and H. T. C. Stoof, Phys. Rev. A 46, R1167 (1992).

[18] W. Thompson and S. Hanrahan, J. Vac. Sci. Technol. 14, 643 (1977); G. Gabrielse, X. Fei, L. A. Orozco, R. L. Tjoelker, J. Haas, H. Kalinowsky, T. A. Trainor, and D. W. Kells, Phys. Rev. Lett. 65, 1317 (1990).

[19] J. P. Hobson, J. Vac. Sci. Technol. 10, 73 (1973).

[20] C. Benvenuti, J. Vac. Sci. Technol. 11, 591 (1974).

[21] D. Sesko, T. Walker, C. Monroe, A. Gallagher, and C. Wieman, Phys. Rev. Lett. 63, 961 (1989).

[22] R. W. Anderson, J. Chem. Phys. 60, 2680 (1974).

[23] C. R. Monroe, Ph.D. thesis, University of Colorado, 1992.

[24] E. W. Rothe and R. B. Bernstein, J. Chem. Phys. 31, 1619 (1959).

[25] K. Helmerson, A. Martin, and D. E. Pritchard, J. Opt. Soc. Am. B 9, 483 (1992).

[26] J. M. Doyle, J. C. Sandberg, I. A. Yu, C. L. Cesar, D. Kleppner, and T. J. Greytak, Phys. Rev. Lett. 67, 603 (1991), and references therein.

[27] J. Weinstein and K. G. Libbrecht (unpublished).

[28] J. T. M. Walraven and I. F. Silvera, Physica 107B, 517 (1981). 